\title{
A Mouse Model for Barrett's Esophagus: Surgery and Histology
}

Taddei $\mathbf{A}^{1 *}$, Lottini $\mathrm{T}^{2}$, Fazi $\mathbf{M}^{1}$, Ringressi $\mathbf{M N}^{1}$, Lastraioli $\mathrm{E}^{2}$, Bechi $\mathbf{P}^{1}$ and Arcangeli $\mathbf{A}^{2}$

${ }^{1}$ Department of Surgery and Translational Medicine, University of Florence, Italy

${ }^{2}$ Department of Experimental and Clinical Medicine, Section of Internal Medicine, University of Florence, Italy

\begin{abstract}
Purpose: Barrett's esophagus (BE) is the sole precursor lesion of esophageal adenocarcinoma (EA) identified so far. The progression towards EA is estimated to affect 2 to $10 \%$ of BE patients, hence endoscopic surveillance of at-risk subjects is mandatory. Surveillance endoscopic procedures imply high cost, discomfort and risks for the patient, as well as the non-infrequent missing of small, focal lesions signaling progression to EA. Hence, it is important to search for new potential markers to better identify BE patients at risk of EA progression. The aim of this study was to produce a mouse model of BE, suitable for further molecular and genetic analyses.
\end{abstract}

Methods: Forty-four CD1 mice were operated upon by means of an esophago-jejunal anastomosis. Five CD1 mice underwent a sham operation. The animals were sacrificed 10 months later and histological analysis was performed with Hematoxylin \& Eosin and Alcian Blue staining.

Results: The overall postoperative mortality rate was $11 \%$. Of the 39 operated animals $14 \%$ developed histologically detectable intestinal metaplasia in the lower esophagus. No histologically detectable lesions were shown in the sham group.

Conclusions: The mice model we propose could be applied because of its technical feasibility and acceptable mortality and can be used in transgenic mice too, in order to better understand molecular progression from BE to esophageal adenocarcinoma.

Keywords: Barrett's esophagus; Mouse model; Intestinal metaplasia; Histology

\section{Introduction}

Barrett's Esophagus (BE) is a condition where the normal squamous epithelium of the esophagus is replaced by intestinal-type columnar epithelium. BE diagnosis is based on the presence of goblet cells of the intestinal type within columnar epithelium [1]. BE is thought to be a precursor lesion for esophageal adenocarcinoma (EA). Despite the relatively low progression rate of $\mathrm{BE}$ towards $\mathrm{EA}$ (EA is estimated to affect 2 to $10 \%$ of $\mathrm{BE}$ patients), endoscopic surveillance of atrisk subjects is commonly applied. However, routinary endoscopic procedures must be applied with caution due to their cost, discomfort and risks for the patient, as well as for the non-infrequent missing of small, focal lesions signaling progression to EA. Considering that $\mathrm{BE}$ incidence has been sharply increasing in the last decades in Western countries [2] and that prognosis is still poor despite of recent advances in treatment [3], it is urgent to decipher the molecular mechanisms underlying $\mathrm{BE}$ progression towards EA, in order to improve its diagnosis and treatment. In particular, it is important to search for new potential markers to better identify BE patients at risk for progression to EA.

For this purpose, several animal models have been developed, however none of them is devoid of some weakness [4]. Indeed, the ideal animal model should encompass all the steps between BE and EA, also modelling the chronic inflammation which underlies any step of esophageal carcinogenesis [5]. Moreover, to better model human BE, genetic heterogeneity should be taken into account for the validation of the animal model [6]. The first animal model for BE was proposed by Bremner in 1970 and it was developed in dogs [7]. In the 90s, several animal models were proposed; however, most of them are rat models while only a few mouse models have been proposed more recently. Mice models are progressively substituting the rat, which is up to now the most used animal for BE models [8]. Indeed, a mouse model for EA, based on a surgical approach, has been recently described [9].
Mouse models usefulness in cancer research is due to several features, in particular mouse genome has been completely sequenced [10] and genetic manipulation is feasible.

The aim of this paper was that of developing and evaluating a surgical mouse model for BE, which could further apply to genetic and pharmacological studies.

\section{Materials and Methods}

\section{$\mathrm{BE}$ induction in mice}

Experiments in CD1 mice were performed at the Laboratory of Genetic Engineering for the Production of Animal Models (L.I.Ge.M.A) at the Animal House of the University of Florence. 44 three-weekold CD1 mice were studied, represented by 39 operated according to the experimental model and 5 control (sham operated) mice at four different times. The characteristics of each series of mice are given in Table 1. Water and a standard chow were given until the day before surgery. Water was discontinued the morning of the operation. Mice were warmed by a hot lamp before and during the operation in order to prevent postoperative hypothermia. Anesthesia was performed by an intraperitoneal injection of Avertin $(17 \mu \mathrm{l} / \mathrm{g})$. All mice were operated on a small operating table and a $2-3 \mathrm{~cm}$ median laparotomy was

*Corresponding author: Antonio Taddei, Department of Surgery and Translational Medicine, University of Florence, Italy, Tel: +393473603797; Fax: +390554220133; E-mail: antonio.taddei@unifi.it

Received June 09, 2017; Accepted August 16, 2017; Published August 21, 2017

Citation: Taddei A, Lottini T, Fazi M, Ringressi MN, Lastraioli E, et al. (2017) A Mouse Model for Barrett's Esophagus: Surgery and Histology. J Carcinogene Mutagene 8: 304. doi: 10.4172/2157-2518.1000304

Copyright: (C) 2017 Taddei A, et al. This is an open-access article distributed under the terms of the Creative Commons Attribution License, which permits unrestricted use, distribution, and reproduction in any medium, provided the original author and source are credited. 
Citation: Taddei A, Lottini T, Fazi M, Ringressi MN, Lastraioli E, et al. (2017) A Mouse Model for Barrett's Esophagus: Surgery and Histology. J Carcinogene Mutagene 8: 304. doi: 10.4172/2157-2518.1000304

performed. After a wide divarication of both left liver lobe and left part of median lobe, esophagogastric junction was exposed. After sectioning of left gastric vessels, distal esophagus was sectioned after clamping in order to avoid esophageal retraction and the gastric side stitched with Goretex 8/0. Jejunum was prepared $5 \mathrm{~cm}$ after Treitz ligament and a small hole was performed in its wall by a $14 \mathrm{Fr}$ intravenous cannula and an end to side esophago-jejunal anastomosis was performed either with Goretex 8/0 or silk 8/0 with the cut end of the esophagus. Abdominal wall was closed with nylon $4 / 0$. As regard as the 5 control mices, a sham operation was performed. The same anesthesia protocol was applied as the other group and the operation consist in opening the abdomen and after an exploration excluding other concomitant pathologies, in closing it with nylon $4 / 0$ as the other group. Liquid feeding was restored in the first post-operative day and solid feeding started the day after. All the animals which got sick or underwent a relevant weight loss were killed. In the control group mice a sham operation was performed. The experiment was ended after 10 months and mice were sacrificed. The study was conducted after the approval of the Ethical Committee, in accordance to the criteria outlined in the Guide for the Care and Use of Laboratory Animals.

\section{Histological analysis}

Samples were taken in the operating room of the animal facility immediately after the animal sacrifice. The entire stomach and esophagus were removed and were immediately fixed in formalin for at least 24 hours. Subsequently, samples were processed for paraffin embedding and $7 \mu \mathrm{m}$ sections were obtained through a microtome and put on positive charged slides. Sections were then stained with Hematoxylin \& Eosin following a standardised protocol and observed under a light microscope. The same samples were stained with Alcian Blue to detect goblet cells presence and an accurate examination of the sections was performed.

\section{Results}

Data reported in this paper were obtained performing an esophagojejunal anastomosis in CD1 mice to establish a mouse model for BE.

Gastrojejunal anastomosis was performed with Goretex 8/0 in 20 mice and with silk $8 / 0$ in 19 mice in order to identify the best surgical technique. No significant difference was found neither for manual ability requested nor for results in terms of anastomotic leakage. A drawing showing the surgical procedure is reported in Figure 1A, while a representative picture showing the result of the surgical procedure is reported in Figure 1B.

Due to technical problems related to bleeding and/or anastomosis, the overall mortality rate was $11 \%$. In particular, since in a first series animals suffered from hypothermia and anaesthesia problems, thereafter in addition to changes in the anaesthetic treatment, the temperature in the operation room was set at $30^{\circ} \mathrm{C}$ and animals were kept at the same temperature for 2 hours after surgery. In the third series survival rate was lower; this was probably due to the lower mean weight of the animals. In the last group a thermophore set at $37^{\circ} \mathrm{C}$ was used, in addition to the previous adjustments and survival rate was significantly higher (Table 1 ). The higher survival rate in the last group of animals was probably due to different reasons: 1) the temperature was kept at higher 7 values to avoid hypothermia; 2) low doses of $2.5 \%$ Avertin were used for anaesthesia; 3 ) animals were fed with semiliquid food, obtained by homogenizing standard food with water, during the first three days after surgery.

Macroscopic evaluation, further corroborated by histological analysis of the samples, showed that animals belonging to the control group did not develop lesions, as witnessed by the presence of an intact Z-line to make the boundary between the squamous and gastric-type epithelia (Figure 2A). All the animals of the operated group showed the signs of gastric mucosal atrophy due to the esophago-jejunal
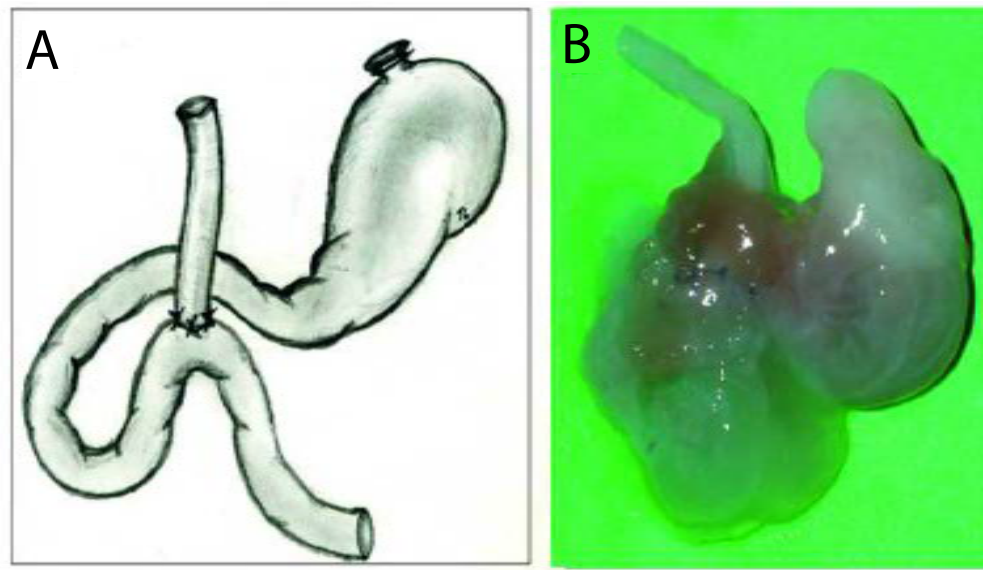

Figure 1: Surgical procedure. A) Drawing of the esophago-jejunal anastomosis performed in this study. B) Representative specimen from an operated animal, showing esophago-jejunal anastomosis

\begin{tabular}{|c|c|c|c|c|c|}
\hline \multirow{2}{*}{ Group } & Number of Animals & Mean Weight (Range) & \multicolumn{2}{|c|}{ Mortality Rate (\%) } \\
\cline { 2 - 5 } & 5 & $28 \mathrm{~g} \mathrm{(26-30)}$ & 0 & 0 \\
\hline I & 15 & $29 \mathrm{~g} \mathrm{(27-31)}$ & 20 & 26.7 \\
\hline III & 12 & $24 \mathrm{~g} \mathrm{(21-26)}$ & 16.7 & 58.3 \\
\hline IV & 12 & $30 \mathrm{~g} \mathrm{(28-34)}$ & 8.3 & 16.7 \\
\hline
\end{tabular}

Table 1: Characteristics and mortality rates of the different groups of animals. 

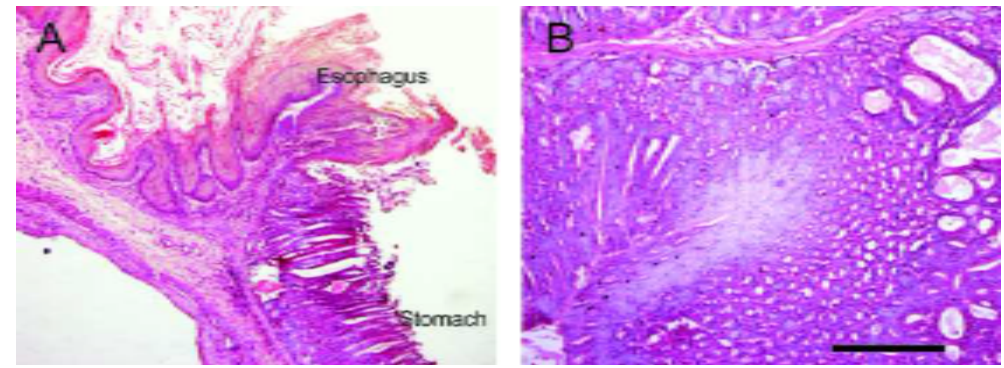

Figure 2: Hematoxylin\&Eosin staining. A) Gastro-Esophageal junction of a control animal. Bar: $200 \mu m$. B) Gastric atrophy in an operated mouse. Bar: $100 \mu \mathrm{m}$
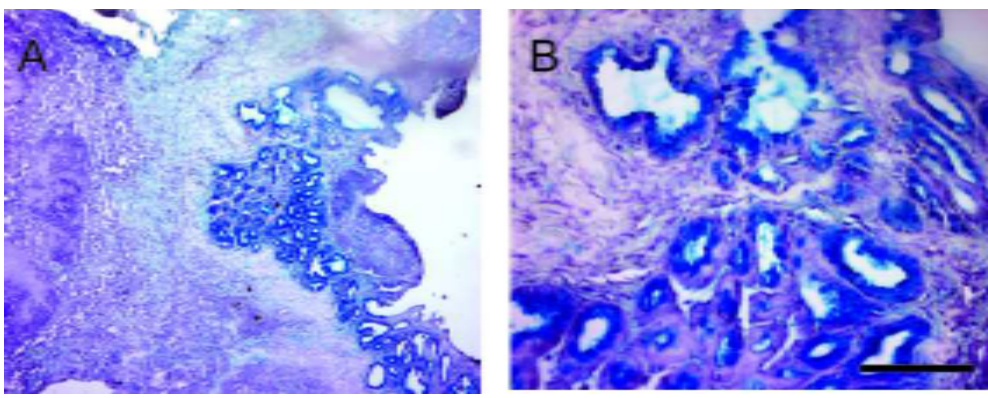

Figure 3: Alcian Blue staining. A) Intestinal metaplasia in an operated mouse, showing the histological signs of BE. Bar: $200 \mu$ m. B) Higher magnification of the Alcian Blue-positive area. Bar: $50 \mu \mathrm{m}$

anastomosis (Figure 2B). Moreover, 6 (14\%) of the operated animals developed histologically detectable intestinal metaplasia in the lower esophagus. On the contrary, none of the controls animal developed atrophy or intestinal metaplasia in the lower esophagus.

When the presence of intestinal metaplasia was suspected, Alcian Blue staining was also performed, in order to detect goblet cells. The picture in Figure 3A shows the presence, at the gastroesophageal junction, of an area characterised by intestinal metaplasia. In Figure 3B a higher power microphotograph is reported, in which goblet cells can be better identified, stained in blue due to the presence of acid mucin which were selectively stained by Alcian Blue dye.

\section{Discussion}

In the past years, many progresses have been made in understanding the mechanisms of reflux esophageal injury which leads to BE and Barrett's adenocarcinogenesis. However, human studies concerning the pathogenesis and relationship between the different steps of the carcinogenetic model obtain different results. It is well known that gastroesophageal reflux (GER) often causes peptic injury and inflammation of the esophageal squamous epi- thelium, a condition called reflux esophagitis. Esophagitis in most cases heals through the regeneration of squamous cells in the distal oesophagus. Conversely, in some patients esophagitis heals through a columnar-metaplastic process, which seems to represent a temptatively protective reaction to reflux injury since the metaplastic epithelium is more resistant to the noxious agents than the native tissue. In a minority of these cases intestinal-type columnar cells are represented; this condition is called Barrett's esophagus (BE) and represents the most common esophageal pre- cancerous lesion. Therefore, the sequence of eventsreflux-esophagitis-intestinal metaplasia (BE)-dysplasia-invasive cancer is widely accepted as the main carcinogenetic pathway in the esophagus. However the mechanisms that underlie this progression need to be defined. Despite many molecules have been widely studied such as COX-2 [11], Herg1 [12], bcl-2 and bcl-xL [13], no conclusive studies are present literature. This is partly due to inter individual variability in humans and to the difficulties both in surveillance and in the interpretation of the endoscopic and histologic findings. For these reasons, the creation of a reproducible experimental model for $\mathrm{BE}$ (reviewed in [5]) is needed and can be useful to better understand the progression from $\mathrm{BE}$ to esophageal adenocarcinoma.

It is well known that, whereas humans might spontaneously develop $\mathrm{BE}$, mice and rats do not, mainly because of differences in esophageal biology. For these reasons, surgery has been generally required for $\mathrm{BE}$ occurrence in rats and mice, although BE might be observed in animals treated with carcinogens, after esophagitis development [8]. Moreover, for the study of the molecular events involved in the carcinogenesis of Barrett's carcinoma, a mouse model would be much more promising since most of the genetically altered animals are mice. However, no such mice models exist nowadays; it is probably due to the high mortality involved with the surgical procedure to create a mixed duodenogastric reflux and with the uncertain postoperative course of mice.

In a recent paper, a surgical approach similar to the one described in this paper was used [9]. However, several differences can be found between the two studies. First, mice included in the present study are 3 weeks old while in the cited paper are 6-8 weeks old. Moreover some differences could be found in surgical technique too, especially in the materials used for sutures, and in the food administration. Finally we have a control group which is necessary to demonstrate the effect of duodenal reflux in the development of $\mathrm{BE}$.

\section{Conclusion}

The aim of the study was to demonstrate that the model we proposed could be applied in mice because of a technical feasibility 
Citation: Taddei A, Lottini T, Fazi M, Ringressi MN, Lastraioli E, et al. (2017) A Mouse Model for Barrett's Esophagus: Surgery and Histology. J Carcinogene Mutagene 8: 304. doi: 10.4172/2157-2518.1000304

and an acceptable mortality. In the literature many surgical procedure and the use of carcinogenic drugs have been reported. In spite of all the limitations of the present paper, our results show that this model can be used to study the development of reflux-induced esophagitis, $\mathrm{BE}$ and EA without using any drug. Therefore this model can be proposed also in engineered mice in order to better understand the role of duodenogastroesophageal reflux as well as the role of molecular markers in EA pathogenesis.

\section{Acknowledgments}

This work was supported by Regione Toscana (D.G.R. 1157/2011 "A novel endoscopic surveillance protocol in patients with Barrett's esophagus to predict the progression to esophageal adenocarcinoma" C.U.P. B11J12000940002) to AA.

\section{References}

1. Wang KK, Sampliner RE, Practice Parameters Committee of the American College of Gastroenterology (2008) Updated guidelines 2008 for the diagnosis, surveillance and therapy of Barrett's esophagus. Am J Gastroenterol 103: 788797.

2. Pohl H, Sirovich B, Welch HG (2010) Esophageal adenocarcinoma incidence: are we reaching the peak? Cancer Epidemiol Biomarkers Prev 19: 1468-1470.

3. Siegel R, Naishadham D, Jemal A (2012) Cancer statistics, 2012. CA Cancer J Clin 62: 10-29.

4. Attwood SE, Harrison LA, Preston SL, Jankowski JA (2008) Esophageal adenocarcinoma in "mice and men": back to basics! Am J Gastroenterol 103: 2367-2372.
5. Garman KS, Orlando RC, Chen X (2012) Review: Experimental models for Barrett's esophagus and esophageal adenocarcinoma. Am J Physiol Gastrointest Liver Physiol 302: G1231-1243.

6. Pavolv K, Maley CC (2010) New models of neoplastic progression in Barrett's oesophagus. Biochem Soc Trans 38: 331-336.

7. Bremner CG, Lynch VP, Ellis FH Jr (1970) Barrett's esophagus: congenital or acquired? An experimental study of esophageal mucosal regeneration in the dog. Surgery 68: 209-216.

8. Xu X, LoCicero J 3rd, Macri E, Loda M, Ellis FH Jr (2000) Barrett's esophagus and associated adenocarcinoma in a mouse surgical model. J Surg Res $88: 120-124$

9. Raggi M, Langer R, Feith M, Friess H, Schauer M, Theisen J (2010) Successful evaluation of a new animal model using mice for esophageal adenocarcinoma. Langenbecks Arch Surg 395: 347-350.

10. Gunter C, Dhand R (2002) The mouse genome. Nature 420: 509.

11. Taddei A, Fabbroni V, Pini A, Lucarini L, Ringressi MN, et al. (2014) Cyclooxygenase-2 and Inflammation Mediators Have a Crucial Role in RefluxRelated Esophageal Histological Changes and Barrett's Esophagus. Dig Dis Sci 59: 949-957.

12. Lastraioli E, Taddei A, Messerini L, Comin Ce, Festini M, et al. (2013) hERG1 Channels in Human Esophagus: Evidence for Their Aberrant Expression in the Malignant Progression of Barrett's Esophagus. J Cell Phisiol 209: 398-404

13. Iravani S, Zhang HQ, Yuan ZQ (2013) Modification of insulin-like growth factor 1 receptor, $\mathrm{c}-\mathrm{Src}$, and $\mathrm{Bcl}-\mathrm{XL}$ protein expression during the progression of Barrett's neoplasia. Hum Pathol 34: 975-982 